\title{
RESISTÊNCIA AO ATAQUE DE FUNGOS APODRECEDORES EM PAINÉIS OSB TERMICAMENTE TRATADOS
}

\author{
Rafael Farinassi Mendes ${ }^{1 *}$, Geraldo Bortoletto Júnior ${ }^{2}$, Alencar Garlet ${ }^{3}$, \\ Natalie Ferreira de Almeida ${ }^{2}$, Paula Gabriella Surdi²
}

*Autor para correspondência: rafaelfarinassi@gmail.com

RESUMO: O objetivo foi de avaliar o efeito do pré e pós-tratamento térmico sobre a resistência dos painéis OSB ao ataque de fungos apodrecedores, e comparar essas duas formas de tratamento térmico com algumas variáveis de produção. O delineamento experimental se constituiu de sete tratamentos, com a avaliação de duas temperaturas de pré-tratamento das partículas (200 e $240^{\circ} \mathrm{C}$ ), por um período de 60 minutos; do pós-tratamento térmico dos painéis, produzidos com e sem a aplicação de parafina, sob temperatura de $220^{\circ} \mathrm{C}$ por um período de 12 minutos; e do aumento do teor de adesivo e a aplicação de parafina em painéis sem tratamento térmico. Para cada tratamento, foram produzidos três painéis com densidade nominal de $0,65 \mathrm{~g} / \mathrm{cm}^{3} \mathrm{e}$ adesivo à base de fenol-formaldeído. Mediante a análise dos resultados foi possível concluir que os painéis com pré-tratamento térmico a $240^{\circ} \mathrm{C}$ apresentaram-se com grande potencial de inibição ao ataque dos fungos Trametes versicolor e Gloeophyllum trabeum, porém, com efeito equivalente ao obtido com o aumento do teor de adesivo fenol-formaldeído. O pós-tratamento térmico não proporcionou melhoria significativa para a resistência biológica dos painéis OSB.

Palavras-chave: Painéis de madeira, tratamento térmico, variáveis de produção, biodeterioração.

\section{DECAY FUNGI RESISTANCE IN THERMALLY TREATED OSB PANELS}

ABSTRACT: This study evaluated the effect of pre and post thermal treatment on the resistance of OSB panels to attack by decay fungi, and compared these two forms of thermal treatment with some production variables. The experimental design consisted of seven treatments, with the evaluation of two temperatures of pre thermal treatment of the particles $\left(200\right.$ and $\left.240{ }^{\circ} \mathrm{C}\right)$ for a 60 minutes period; post thermal treatment of the panels, produced with and without the application of paraffin, at $220^{\circ} \mathrm{C}$ of temperature for a period of 12 minutes; and increasing the content of adhesive and application of paraffin in panels without thermal treatment. For each treatment three panels were produced with nominal density of $0.65 \mathrm{~g} / \mathrm{cm}^{3}$ and phenol-formaldehyde adhesive. By analyzing the results it was concluded that the panels with pre thermal treatment at $240{ }^{\circ} \mathrm{C}$ presented great potential for inhibiting the attack of Trametes versicolor fungi and Gloeophyllum trabeum, but with equivalent effect to that obtained with the increasing the content phenol-formaldehyde adhesive. The post thermal treatment did not increase significantly the biological resistance of the OSB panels.

Key words: Wood panels, thermal treatment, production variables, biodeterioration.

\section{INTRODUÇÃO}

Atualmente, o setor de painéis de madeira tem apresentado grande crescimento no Brasil e no Mundo. No Brasil, isso se deve em função de fatores como a modernização do parque fabril, o surgimento de novos produtos e/ou melhorias de painéis do tipo Oriented Strand Board (OSB), Medium Density Fiberboard (MDF), High Density Fiberboard (HDF), Medium Density Particleboard (MDP) e High Density Particleboard (HDP), além do aumento da demanda dos setores de construção civil e de móveis, os quais são os principais consumidores de painéis de madeira (MENDES, 2011).

Com essa crescente demanda pelos diferentes tipos de painéis reconstituídos, torna-se primordial a melhoria da qualidade desses produtos. Assim, o conhecimento acerca da resistência de painéis, à base de materiais lignocelulósicos, ao ataque de microrganismos deve servir de base para a prevenção da degradação e a correta destinação para o uso do material (OKINO, 2007). Principalmente no caso dos painéis OSB, os quais são utilizados como elemento estrutural e não podem apresentar diminuição de suas resistências mecânicas, em decorrência da degradação biológica.

O tratamento térmico da madeira tem ganhado destaque como um método eficaz para melhorar sua estabilidade dimensional e resistência biológica frente a organismos xilófagos (BOURGOIS; GUYONNET, 1988; HILLIS, 1984; HOMAN et al., 2000; MOMOHARA et al., 2003; ROUSSET et al., 2004; STAMM, 1956). Sendo atualmente utilizado em escala industrial na Finlândia, França, Holanda e Alemanha, para o tratamento da madeira maciça (ROUSSET et al., 2004).

\footnotetext{
${ }^{1}$ Universidade Federal de Lavras - Lavras, Minas Gerais, Brasil

${ }^{2}$ Escola Superior de Agricultura Luiz de Queiroz - Piracicaba, São Paulo, Brasil

${ }^{3}$ Serviço Florestal Brasileiro - Brasília, Distrito Federal, Brasil
}

Cerne, Lavras, v. 19, n. 4, p. 551-557, out./dez. 2013 
As alterações na composição química da madeira tratada termicamente, e também de seus produtos, podem explicar muitas das alterações observadas em diversas de suas propriedades. Assim, quando a madeira é aquecida, ocorrem mudanças na natureza de seus componentes (celulose, hemicelulose, lignina e extrativos), sobretudo as hemiceluloses que são, dentre eles, os mais sensíveis à ação do calor, o que pode modificar sua higroscopicidade, estabilidade dimensional, permeabilidade e resistência biológica (BYRNE; NAGLE, 1997; HILLIS, 1984).

Com os resultados expressivos obtidos com a aplicação do tratamento térmico para a madeira maciça, algumas pesquisas relativamente recentes vêm sendo desenvolvidas sobre o tratamento térmico de painéis OSB, sob dois modos distintos: pós e pré-tratamento. A diferença básica entre essas duas formas deve-se ao fato de que o pré-tratamento térmico é efetuado na matéria-prima dos painéis, ou seja, nas partículas, e o pós-tratamento térmico é efetuado nos painéis, depois de consolidados e estabilizados.

Os resultados também se mostraram bastante promissores quanto à estabilidade dimensional dos painéis OSB, tanto no pós como no pré-tratamento térmico (DEL MENEZZI, 2004; DEL MENEZZI; TOMASELLI, 2006; MENDES, 2011). Contudo, quanto à ação do tratamento térmico sobre a resistência biológica dos painéis OSB, poucos são os estudos realizados, tanto no pós-tratamento térmico (DEL MENEZZI et al., 2008; SOUZA; DEL MENEZZI, 2006) como no pré-tratamento térmico (PAUL et al., 2007). Neste contexto, com o presente estudo, objetivou-se avaliar o efeito do pré e pós-tratamento térmico sobre a resistência dos painéis OSB ao ataque de fungos, assim como da comparação dessas duas formas de tratamento térmico com algumas variáveis de produção.

\section{MATERIAL E METODOS}

\subsection{Material de estudo}

Os painéis OSB foram produzidos com a madeira de Pinus taeda L., aos 35 anos de idade e com densidade básica média de $0,473 \mathrm{~g} / \mathrm{cm}^{3}$.

Outros parâmetros adotados para a produção dos painéis foram as partículas strand com dimensões de $0,65 \times 25 \times 90 \mathrm{~mm}$ (espessura, largura e comprimento, respectivamente); composição em três camadas, cujas proporções de partículas, em massa, foram de 30:40:30 (face, miolo e face, respectivamente), densidade nominal de $0,65 \mathrm{~g} / \mathrm{cm}^{3}$ e adesivo fenol-formaldeído.

Cerne, Lavras, v. 19, n. 4, p. 551-557, out./dez. 2013
O delineamento experimental se constituiu de sete tratamentos, conforme descrito na Tabela 1. Para cada um dos tratamentos foram produzidos três painéis OSB.

Tabela 1 - Delineamento experimental para a produção dos painéis OSB.

Table 1 - Experimental design for the production of OSB panels.

\begin{tabular}{ccccc}
\hline Tratamentos & $\begin{array}{c}\text { Tratamento } \\
\text { térmico das } \\
\text { partículas }\end{array}$ & $\begin{array}{c}\text { Tratamento } \\
\text { térmico dos } \\
\text { painéis OSB }\end{array}$ & $\begin{array}{c}\text { Teor de } \\
\text { adesivo } \\
(\%)\end{array}$ & $\begin{array}{c}\text { Teor de } \\
\text { Parafina } \\
(\%)\end{array}$ \\
\hline T1 & - & - & 6 & - \\
T2 & $200^{\circ} \mathrm{C}$ & - & 6 & - \\
T3 & $240^{\circ} \mathrm{C}$ & - & 6 & - \\
T4 & - & $220^{\circ} \mathrm{C} / 12 \mathrm{~min}$ & 6 & - \\
T5 & - & - & 6 & 1 \\
T6 & - & - & 8 & - \\
T7 & - & $220^{\circ} \mathrm{C} / 12 \mathrm{~min}$ & 6 & 1 \\
\hline
\end{tabular}

Esse delineamento se constituiu da avaliação de três métodos que visavam ao aumento da resistência a biodeterioração a fungos, sendo estes: diferentes variáveis de produção (aumento do teor de adesivo e aplicação de parafina); pré-tratamento térmico nas partículas strand nas temperaturas de $200^{\circ} \mathrm{C}$ e $240^{\circ} \mathrm{C}$ por período de 60 minutos; e pós-tratamento térmico aplicado ao painel OSB na temperatura de $220^{\circ} \mathrm{C}$ por período de 12 minutos. Os procedimentos do pré e pós-tratamento térmico foram realizados conforme trabalho de Mendes (2011).

\subsection{Preparação dos corpos de prova}

O ensaio de apodrecimento acelerado foi realizado na Área de Biodegradação e Preservação da Madeira, do Laboratório de Produtos Florestais (LPF) / Serviço Florestal Brasileiro, em Brasília, DF. Utilizou-se a metodologia proposta pela norma ASTM D2017 (AMERICAN SOCIETY FOR TESTING AND MATERIALS - ASTM, 2005).

Para tanto, os corpos de prova foram preparados nas dimensões de $25 \times 25 \mathrm{~mm} \times$ espessura do painel (comprimento, largura e espessura, respectivamente). Foram ensaiados 12 corpos de prova (4 por painel) por tratamento para cada fungo. Para os blocos de correção foram utilizados seis corpos de prova por tratamento.

Todos os corpos de prova foram acondicionados em estufa com circulação forçada de ar a $50^{\circ} \mathrm{C}$, até 
atingir massa constante. Posteriormente, foram pesados em balança analítica, com resolução de $0,0001 \mathrm{~g}$, e o resultado registrado como Mi (massa inicial). Após a pesagem inicial, os corpos de prova foram esterilizados em autoclave à $121^{\circ} \mathrm{C}$ por 30 minutos.

\subsection{Fungos xilófagos}

Foram empregadas duas espécies de fungos xilófagos provenientes da coleção do LPF: Trametes versicolor (Linnaeus ex Fries) Pilat, espécie causadora de podridão branca, e Gloeophyllum trabeum (Persoon. ex Fries.) Murr., espécie causadora de podridão parda.

Os fungos foram repicados em meio de cultura malte líquido à $3 \%$, e levados à incubadora $\left(26 \pm 1^{\circ} \mathrm{C}\right.$ e $70 \pm 5 \%$ de umidade relativa), até o micélio recobrir totalmente a superfície do meio (três semanas). No momento da inoculação, o micélio foi fragmentado com liquidificador doméstico e, em seguida, pipetado para os frascos de ensaio.

Todas as operações de manipulação de fungos (repicagem, inoculação, fragmentação do micélio, introdução de corpos de prova nos frascos de ensaio) foram realizadas sob condições assépticas, no interior de capela de fluxo laminar.

\subsection{Ensaio de apodrecimento}

Foram usados frascos de vidro transparente, com boca larga, tampa rosqueável e capacidade de $250 \mathrm{~mL}$, contendo $100 \mathrm{~g}$ de solo (horizonte B de solo podzólico vermelho-amarelo), livre de matéria orgânica. O solo recebeu adição prévia de calcário, para elevar o $\mathrm{pH}$ a 6,0. A umidade do solo foi ajustada para $130 \%$ da capacidade de campo.

Em cada frasco, foi colocada uma placa suporte de $33 \times 29 \times 3 \mathrm{~mm}$ para o desenvolvimento inicial do fungo. Foram utilizadas placas suporte de pinus (Pinus sp.), para o cultivo de Gloeophyllum trabeum; e, placas de embaúba (Cecropia sp.) para o cultivo de Trametes versicolor. Os frascos foram autoclavados a $121^{\circ} \mathrm{C}$ por 45 minutos. Cada frasco foi inoculado com $1 \mathrm{ml}$ de meio de cultura, contendo o micélio fragmentado, depositado diretamente sobre a placa suporte.

Após a inoculação os frascos foram levados à incubadora, com temperatura de $26 \pm 1^{\circ} \mathrm{C}$ e $70 \pm 5 \%$ de umidade relativa, até o micélio cobrir totalmente a placa suporte ( 2 semanas). Após a colonização das placas suporte, colocou-se um corpo de prova em cada frasco de ensaio.
As amostras permaneceram em contato com os fungos por 12 semanas na câmera climática em condições já descritas.

Encerrado o período de apodrecimento, os corpos de prova foram retirados dos frascos de ensaio e submetidos a uma limpeza cuidadosa para a remoção do excesso de micélio aderido. Posteriormente, foram condicionados em estufa, sob as mesmas condições préensaio. Após atingirem massa constante, foram novamente pesados para determinação da massa final (Mf).

\subsubsection{Blocos de correção}

Para cada tratamento, seis corpos de prova foram introduzidos em frascos de ensaio não inoculados (sem fungos) e receberam o mesmo tratamento e manipulação dos demais. Os blocos de correção têm o objetivo de determinar a perda de massa resultante da manipulação dos corpos de prova, não causada pela ação dos fungos.

\subsubsection{Espécies de referência}

Foram utilizados 18 corpos de prova $(25 \times 25 \times$ $9 \mathrm{~mm}$ ) de pinus (Pinus sp.) como espécie de referência para o fungo G. trabeum e 18 corpos de prova de embaúba (Cecropia sp.) para o fungo T. versicolor. O ensaio foi encerrado, quando a perda de massa média da espécie referência foi superior a $50 \%$.

Os corpos de prova de referência são indicadores do vigor vegetativo da cultura fúngica utilizada no ensaio e das condições de incubação. Segundo a norma ASTM D 2017 (ASTM, 2005), o teste é considerado válido somente quando o fungo provoca perda de massa de, no mínimo, $50 \%$ sobre a espécie de referência, num período de 12 até 16 semanas.

A seleção das espécies de referência levou em consideração a existência de dados de vários ensaios realizados no LPF, que comprovam serem essas espécies susceptíveis aos fungos utilizados.

\subsection{Determinação da perda de massa}

A partir dos valores de massa inicial e final, foi calculada a perda de massa dos corpos de prova conforme a equação 1 .

$\operatorname{Pmt}(\%)=((\mathrm{Mi}-\mathrm{Mf}) /(\mathrm{Mi})) \times 100 \quad$ (Equação 1)

Onde: $\mathrm{Pmt}=$ perda de massa total

$\mathrm{Mi}=$ massa inicial

$\mathrm{Mf}=$ peso final

Cerne, Lavras, v. 19, n. 4, p. 551-557, out./dez. 2013 
Com os valores de perda de massa total e perda de massa dos blocos de correção, foi calculada a perda de massa corrigida, conforme a equação 2 .

Pmc $(\%)=$ Pmt - Pmbc

(Equação 2)

Onde: Pmc $=$ Perda de massa corrigida

Pmt $=$ Média de perda de massa total

Pmbc = Média de perda de massa dos blocos de correção do tratamento

Com base na média da perda de massa corrigida, os tratamentos foram classificados quanto à resistência ao ataque dos fungos, segundo os critérios estabelecidos da ASTM D 2017 (ASTM, 2005), apresentado na Tabela 2.

Tabela 2 - Classes de resistência ao apodrecimento segundo a norma ASTM D2017 (ASTM, 2005).

Table 2 - Resistance classes to decay according to ASTM D2017 (ASTM, 2005).

\begin{tabular}{lcc}
\hline Classe de Resistência & $\begin{array}{c}\text { Perda de } \\
\text { massa (\%) }\end{array}$ & $\begin{array}{c}\text { Massa } \\
\text { residual (\%) }\end{array}$ \\
\hline Alta Resistência (AR) & $0-10$ & $90-100$ \\
Resistente (R) & $11-24$ & $76-89$ \\
Resistência Moderada (RM) & $25-44$ & $56-75$ \\
Não-Resistente (NR) & $>45$ & $>55$ \\
\hline
\end{tabular}

\subsection{Análise estatística}

Para a análise dos dados foi considerado um experimento em delineamento inteiramente casualizado. A resistência à biodeterioração dos diferentes tratamentos aos fungos Trametes versicolor e Gloeophyllum trabeum foram avaliados por análise de variância e teste de ScottKnott a 5\% de significância.

\section{RESULTADOS E DISCUSSÃO}

A perda de massa observada nas madeiras de referência foi de $50,76 \%$ na madeira de embaúba, causada pelo fungo Trametes versicolor e de 53,33\% na madeira de pinus, causada pelo fungo Gloeophyllum trabeum. Esses valores foram observados após 12 semanas de ensaio e são superiores ao valor mínimo de 50\% estabelecido pela norma ASTM D-2017 (ASTM, 2005). Assim, esse ensaio foi encerrado após 12 semanas de exposição dos corpos de prova aos fungos apodrecedores.

Cerne, Lavras, v. 19, n. 4, p. 551-557, out./dez. 2013
Esses dados atestam que as condições gerais de ensaios foram favoráveis ao crescimento dos fungos e que as culturas empregadas estavam em pleno vigor vegetativo. Portanto, os resultados são válidos de acordo com os critérios da norma. Na avaliação das análises estatísticas, obtidas pelo teste de Scott-Knott $(\alpha=0,05)$, para a perda de massa dos painéis OSB, proporcionado pelo fungo Trametes versicolor (Figura 1) e pelo fungo Gloeophyllum trabeum (Figura 2), observa-se que os painéis produzidos com o pré-tratamento térmico das partículas strand a $240{ }^{\circ} \mathrm{C}$ e também aqueles com o aumento do teor de adesivo para $8 \%$ apresentaram-se iguais estatisticamente e diferentes dos demais tratamentos para os dois tipos de fungos, obtendo, assim, os menores valores médios de perda de massa.

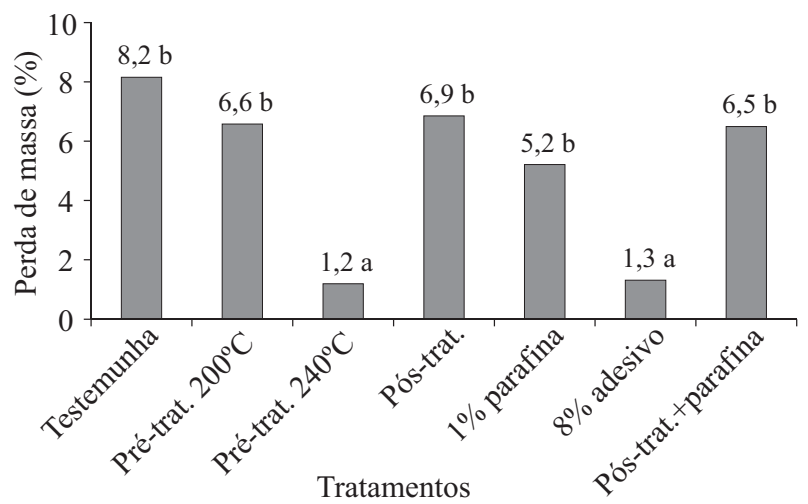

Figura 1 - Perda de massa causada pelo fungo Trametes versicolor.

Figure 1-Mass loss caused by the fungus Trametes versicolor.

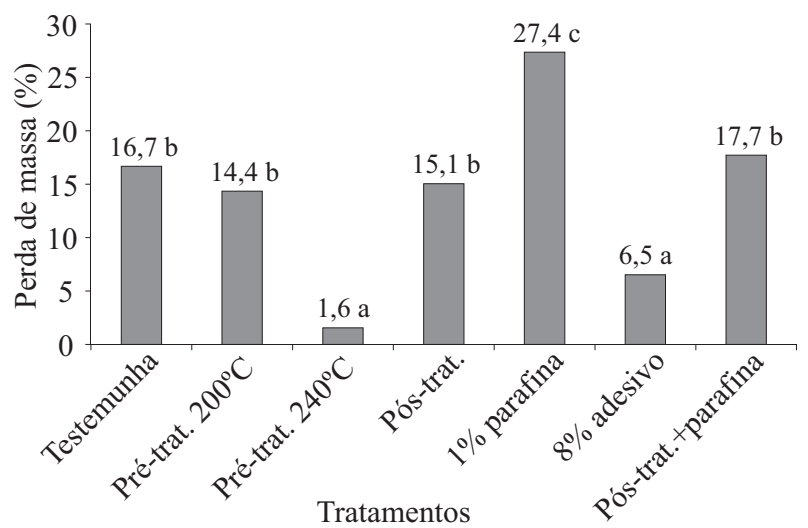

Figura 2 - Perda de massa causada pelo fungo Gloeophyllum trabeum.

Figure 2-Mass loss caused by the fungus Gloeophyllum trabeum. 
Como foi observada diferença estatística entre os painéis testemunha ( $6 \%$ de adesivo fenol-formaldeído) e os painéis produzidos com o aumento do teor de adesivo ( $8 \%$ de adesivo fenol-formaldeído), nos permite concluir que, com o aumento do teor de adesivo, ocorre a diminuição significativa da porcentagem de perda de massa causada por esses dois tipos de fungos. O mesmo foi verificado por Okino (2007), os quais, avaliando a resistência a biodeterioração à fungos de painéis OSB produzidos com a madeira de pinus, eucalipto e cipreste, em diferentes tipos e teores de adesivos, observaram que com o aumento do teor de adesivo de 5\% para $8 \%$ ocorreu redução significativa na perda de massa dos painéis. Okino et al. (2009), estudando a resistência a fungos de painéis OSB produzidos com a madeira de Hevea brasiliensis também observou a diminuição da perda de massa causada pelos fungos Gloeophyllum trabeum e Trametes versicolor com o aumento do teor de adesivo fenol-formaldeído, os autores atribuíram essa melhoria ao potencial biocida dos compostos fenólicos do adesivo.

$\mathrm{Na}$ análise do pré-tratamento térmico, para os dois tipos de fungos, observou-se efeito significativo apenas para a temperatura de $240^{\circ} \mathrm{C}$ em relação aos painéis testemunha, apesar de se notar também certa tendência de melhoria para o pré-tratamento a $200^{\circ} \mathrm{C}$. Paul et al. (2007), avaliando painéis OSB produzidos com partículas tratadas termicamente a temperaturas de 180, 200, 220 e $240^{\circ} \mathrm{C}$, em tempos totais de processo de 30,60 e 90 minutos, também observaram aumento da resistência dos painéis ao ataque de fungos, conforme a elevação da temperatura do tratamento térmico das partículas strand. De acordo com Weiland e Guyonnet (2003), a melhoria da resistência ao ataque de fungos, em consequência do tratamento térmico da madeira, pode estar relacionada com os seguintes fatores: a eliminação das pentosanas, fonte primária de nutrientes, resultando em inibição da invasão inicial; a formação de furfural e a reticulação deste com a trama de lignina remanescente, fazendo com que o sistema enzimático dos fungos perca a habilidade de identificar a madeira como um substrato; e a formação de produtos tóxicos cujas substâncias podem atuar como fungicidas.

No caso do pós-tratamento térmico não foi observada diferença significava em relação aos painéis testemunha para os dois tipos de fungos (Figuras 1 e 2), provavelmente pela degradação menos acentuada dos constituintes químicos da madeira. Mas, apesar disso, foi observada certa tendência da diminuição da perda de massa com a aplicação do pós-tratamento térmico, com exceção dos painéis com pós-tratamento térmico e com a aplicação de parafina na avaliação com o fungo Gloeophyllum trabeum, os quais apresentaram maior valor médio de perda de massa que os painéis testemunha. Contudo, tal efeito pode estar associado ao efeito negativo da aplicação da parafina na resistência dos painéis OSB ao ataque do fungo Gloeophyllum trabeum (Figura 2), visto que se observou diferenciação significativa dos painéis produzidos com $1 \%$ de parafina em relação aos painéis testemunha, sendo os painéis com a aplicação de parafina os que apresentaram a maior perda de massa.

Apesar da diferenciação estatística entre os diferentes tratamentos analisados, na Tabela 3 estão apresentados a classificação desses tratamentos quanto à resistência a perda de massa de acordo com a norma ASTM D2017 (ASTM, 2005).

Para o fungo T. versicolor (podridão branca), todos os tratamentos foram classificados como altamente resistentes. Enquanto que para o fungo G. trabeum (podridão parda), apenas os tratamentos com prétratamento térmico a $240{ }^{\circ} \mathrm{C}$ e com o aumento do teor de adesivo para $8 \%$ tiveram essa classificação, sendo os tratamentos testemunha, pré-tratamento térmico a $200^{\circ} \mathrm{C}$ e ambos os tratamentos com pós-tratamento térmico classificados como resistentes, e o tratamento com a aplicação de $1 \%$ de parafina classificado como moderadamente resistente.

Ainda na análise da Tabela 3, verifica-se que a perda de massa foi superior para podridão parda em todos os tratamentos. Esses resultados são decorrentes da utilização de madeira de conífera $(P$ taeda) para a produção dos painéis, pois, fungos de podridão branca se desenvolvem melhor em madeira de folhosas, enquanto fungos de podridão parda causam maiores perdas de massa em coníferas (CURLING; MURPHY, 2002; MARTÍNEZ et al., 2005).

Para fins de indicação de uso, quando um material ensaiado apresenta classe de resistência diferente para os fungos testados, por critério de segurança, deve-se considerar os resultados do fungo que apresentou a maior perda de massa. Portanto, apenas os tratamentos com prétratamento térmico a $240{ }^{\circ} \mathrm{C}$ e com o aumento do teor de adesivo para $8 \%$ podem ser utilizados em situações onde exista risco de apodrecimento. Os painéis classificados como resistentes podem ser utilizados em situações onde haja risco de umidificação eventual, porém, fora do contato com solo ou água. Materiais classificados como moderadamente resistentes só devem ser usados fora do contato com a umidade, sob pena de serem atacados por fungos. Para esses painéis, são necessários estudos

Cerne, Lavras, v. 19, n. 4, p. 551-557, out./dez. 2013 
Tabela 3 - Classes de resistência dos painéis OSB.

Table 3 - Resistance classes of OSB panels.

\begin{tabular}{lcccc}
\hline \multirow{2}{*}{ Tratamentos } & \multicolumn{2}{c}{ Podridão branca } & \multicolumn{2}{c}{ Podridão parda } \\
\cline { 2 - 5 } & Trametes versicolor & Classe de resistência & Gloeophyllum trabeum & Classe de resistência \\
\hline Testemunha & $8,2 \%(3,1)$ & AR & $16,7 \%(6,7)$ & $\mathrm{R}$ \\
Pré-trat. $200^{\circ} \mathrm{C}$ & $6,6 \%(3,1)$ & AR & $14,4 \%(3,9)$ & $\mathrm{R}$ \\
Pré-trat. $240^{\circ} \mathrm{C}$ & $1,2 \%(0,8)$ & AR & $1,6 \%(0,7)$ & $\mathrm{AR}$ \\
Pós-trat. & $6,9 \%(5,2)$ & AR & $15,1 \%(8,2)$ & $\mathrm{R}$ \\
$1 \%$ parafina & $5,2 \%(3,0)$ & AR & $27,4 \%(6,9)$ & $\mathrm{RM}$ \\
$8 \%$ adesivo & $1,3 \%(1,0)$ & AR & $6,5 \%(4,0)$ & $\mathrm{AR}$ \\
Pós-trat.+parafina & $6,5 \%(5,0)$ & AR & $17,7 \%(8,4)$ & $\mathrm{R}$ \\
Pinus (referência) & - & - & $53,3 \%(10,7)$ & $\mathrm{NR}$ \\
Embaúba (referência) & $50,8 \%(3,6)$ & $\mathrm{NR}$ & - & - \\
\hline
\end{tabular}

$\mathrm{AR}=$ Alta resistência; $\mathrm{R}=$ resistente; $\mathrm{RM}=$ resistência modera; $\mathrm{NR}=$ não resistente, segundo ASTM D2017 (ASTM, 2005). Os valores entre parênteses são os desvios padrões.

da aplicação de produtos preservativos para melhorar sua resistência ao ataque de fungos xilófagos (DIROL; DEGLISE, 2001).

\section{CONCLUSÕES}

Os painéis com pré-tratamento térmico a $240^{\circ} \mathrm{C}$ apresentaram-se com grande potencial de inibição do ataque dos fungos Trametes versicolor e Gloeophyllum trabeum, sendo tal resultado decorrente de alterações químicas sofrida pela madeira.

No entanto, o efeito do pré-tratamento térmico a $240^{\circ} \mathrm{C}$ foi equivalente estatisticamente ao obtido com o aumento do teor de adesivo fenol-formaldeído. Permitindo, assim, ser utilizado qualquer um dos métodos para promover o aumento da resistência biológica dos painéis OSB, e cabendo, nesse caso, uma avaliação econômica do método a ser utilizado.

O pós-tratamento térmico não proporcionou melhoria significativa para a resistência biológica dos painéis OSB.

A aplicação de parafina ocasionou efeito negativo na resistência dos painéis.

\section{REFERÊNCIAS}

AMERICAN SOCIETY FOR TESTING AND MATERIALS. ASTM D-2017: standard method for accelerated laboratory test of natural decay resistance of woods. Annual Book of ASTM Standards, Philadelphia, n. 410, p. 5, 2005.

Cerne, Lavras, v. 19, n. 4, p. 551-557, out./dez. 2013
BOURGOIS, J.; GUYONNET, R. Characterization and analysis of torrified wood. Wood Science and Technology, New York, v. 22, p. 143-155, 1988.

BYRNE, C. E.; NAGLE, D. C. Carbonization of wood for advanced materials applications. Carbon, Elmsford, v. 35, n. 2, p. 259-266, 1997.

CURLING, S. F.; MURPHY, R. J. The use of the Decay Susceptibility Index (DSI) in the valuation of biological durability tests of wood based board materials. Holz als Roh und Werkstoff, Berlin, n. 60, p. 224-226, 2002.

\section{DEL MENEZZI, C. H. S. Estabilização dimensional} por meio do tratamento térmico e seus efeitos sobre as propriedades de painéis de partículas orientadas (OSB). 2004. 226 p. Tese (Doutorado em Engenharia Florestal) Universidade Federal do Paraná, Curitiba, 2004.

DEL MENEZZI, C. H. S.; SOUZA, R. Q.; THOMPSON, R. M.; OKINO, E. Y. A.; COSTA, A. F. Properties after weathering and decay resistance of a thermally modified wood structural board. International Biodeterioration \& Biodegradation, Birmingham, v. 62, n. 4, p. 448-454, 2008.

DEL MENEZZI, C. H. S.; TOMASELLI, I. Contact thermal post-treatment of oriented strandboard to improve dimensional stability: a preliminary study. Holz als Roh- und Werkstoff, Berlin, v. 64, p. 212-217, 2006. 
DIROL, D.; DEGLISE, X. Durabilitè dês bois et problèmes associés. Paris: Hermes Science Europe, 2001. 416 p.

HILLIS, W. E. High temperature and chemical effects on wood stability: part 1, general considerations. Wood Science and Technology, Berlin, v. 18, n. 4, p. 281-293, 1984.

HOMAN, W.; TJEERSDSMA, B.; BECKERS, E.; JORISSEN, A. Structural and other properties of modified wood. In: WORLD CONFERENCE ON TIMBER ENGINEERING, 2000, British Columbia. Proceedings... British Columbia: World Conference Timber Enginnering, 2000. p. 1-8.

MARTÍNEZ, A. T.; SPERANZA, M.; FERREIRA, P.; CAMARERO, S.; GUILLÉN, F.; MARTÍNEZ, M. J.; GUTIÉRREZ, A.; DEL RIO, J. C. Biodegradation of lignocellulosics: microbial, chimical, and enzymatic aspects of the fungal attack of lignin. International Microbiology, Madrid, v. 8, p. 195-204, 2005.

MENDES, R. F. Efeito do tratamento térmico sobre as propriedades de painéis OSB. 2011. 115 p. Dissertação (Mestrado em Engenharia Florestal) - Escola Superior de Agricultura "Luiz de Queiroz”, Piracicaba, 2011.

MOMOHRA, I.; OHMURA, W.; KATO, H.; KUBOJIMA, Y. Effect of high-temperature treatment on wood durability against the Brown-rot fungus, Fomitopsis palustris, and the térmite, Coptotermes formosanus. In: INTERNATIONAL IUFRO WOOD DRYING CONFERENCE, 8., 2003, Kanazawa. Proceedings... Kanazawa: IUFRO, 2003. p. 284-287.

OKINO, E. Y. A. Biodegradacao de chapas de particulas orientadas de pinus, eucalipto e cipreste expostas a quatro fungos apodrecedores. Scientia Forestalis, Piracicaba, n. 74, p. 67-74, 2007.

OKINO, E. Y. A.; ALVES, M. V. S.; TEIXEIRA, D. E.; SOUZA, M. R.; SANTANA, M. A. E.; DEL MENEZZI, C. H. S. Ensaio acelerado de laboratório de chapas OSB de seringueira submetidas ao ataque de fungos apodrecedores.

Ciência Florestal, Santa Maria, v. 19, n. 3, p. 333-341, 2009.

PAUL, W.; OHLMEYER, M.; LEITHOFF, H. Thermal modification of OSB-strands by a one-step heat pre-treatment: influence of temperature on weight loss, hygroscopicity and improved fungal resistance. Holz als Roh- und Werkstoff, Berlin, v. 65, p. 57-63, 2007.

ROUSSET, P.; PERRÉ, P.; GIRARSD, P. Modification of mass transfer properties in poplar wood ( $P$. robusta) by thermal treatment at high temperature. Holz als Roh-und Werkstoff, Berlin, v. 62, n. 2, p. 113-119, 2004.

SOUZA, R. Q.; DEL MENEZZI, C. H. S. Resistência biológica de painéis de partículas orientadas (OSB) tratados termicamente. In: REUNIÃO ANUAL DA SBPC, 58., 2006, Florianópolis. Anais... Florianópolis: UFSC, 2006. 1 CDROM.

STAMM, A. J. Thermal degradation of wood and cellulose. Industrial and Engineering Chemistry, Madison, v. 48, n. 3, p. $413-417,1956$.

WEILAND, J. J.; GUYONNET, R. Study of chemical modifications and fungi degradation of thermally modified wood using DRIFT-spectroscopy. Holz als Roh-und Werkstoff, Berlin, v. 61, p. 216-220, 2003.

Recebido: 9 de agosto de 2011; aceito: 24 de maio de 2013. 
